\title{
Vigilancia de la resistencia a antimicrobianos
}

\author{
PATRICIA GONZÁLEZ A. ${ }^{1}$
}

\author{
SURVEILLANCE OF ANTIMICROBIAL RESISTANCE \\ Key words: Resistance, Antimicrobial agents, Surveillance, Mycobacterium, \\ Neisseria, Haemophilus, Streptococcus pneumoniae.
}

La emergencia de la resistencia bacteriana a antimicrobianos y su diseminación es una de las mayores epidemias del mundo en la actualidad. Es bien conocida la gran capacidad de adaptación que presentan las bacterias frente a medios adversos. Como ejemplo de esto se cuentan los diferentes mecanismos desarrollados por estos agentes frente a los antimicrobianos disponibles en la actualidad y en muchos casos frente a fármacos que aún se encuentran es su etapa de evaluación. La única alternativa que se vislumbra para evitar una progresión de esta situación consiste en aplicar programas efectivos que limiten el uso masivo o inadecuado de los agentes antimicrobianos y de esa forma disminuir la exposición y por lo tanto evitar la presión selectiva que éstos ejercen en el medio.

Un paso esencial, previo a la aplicación de cualquier medida preventiva, es contar con un diagnóstico de la situación presente y con un sistema de vigilancia de la tendencia de la resistencia en el tiempo, lo que permitirá evaluar las diferentes políticas adoptadas.

Una red de vigilancia de resistencia a antimicrobianos reúne los propósitos antes mencionados, ya que permite detectar patógenos emergentes, investigar los factores que influyen en la emergencia de resistencia y facilita la elaboración de estrategias de control y su evaluación. Cabe señalar que estos objetivos sólo se logran en su totalidad si la red de vigilancia cuenta con una metodología válida y común a todos los participantes y además con una adecuada selección de la muestra y riguroso análisis, lo que permitirá aplicar en forma correcta los resultados obtenidos.

En nuestro país la vigilancia de la resistencia a antimicrobianos se ha realizado principalmente asociada a los distintos programas de prevención y control de cierta enfermedades infecciosas. En general la vigilancia en Chile se ha realizado mediante una recolección y análisis centralizado en el Instituto de Salud Publica de Chile, ente encargado de analizar y difundir estos resultados

Entre los patógenos sujetos a vigilancia de resistencia se encuentran los siguientes:

- Mycobacterium tuberculosis

- Neisseria gonorrhoeae

- Patógenos meníngeos: Haemophilus influenzae b. Streptococcus pneumoniae, Neisseria meningitidis

- Agentes de importancia en infecciones intrahospitalarias

\section{Mycobacterium tuberculosis}

La vigilancia de este agente se realiza en forma permanente desde los inicios del programa de control de la tuberculosis, a mediados de

${ }^{1}$ Laboratorio de Microbiología, Hospital Dr. Sótero del Río. 
la década de los 60. Consiste básicamente en el estudio prospectivo de cohortes de pacientes recién diagnosticados en los cuales se evalúa el comportamiento de cepas frente a diferentes agentes antituberculosos. Los niveles de resistencia inicial (pacientes nunca tratados) como de resistencia adquirida (pacientes con recaída o fracaso de tratamiento) en distintos años se muestran en la Tabla 1.

Se aprecia que la resistencia inicial no ha variado en el tiempo, manteniéndose en niveles bajos, siendo estreptomicina el fármaco que presenta el mayor porcentaje de resistencia.

La resistencia adquirida muestra una mayor frecuencia a agentes de primera línea, la cual ha tendido a disminuir en el tiempo. Cabe destacar el bajo porcentaje de multiresistencia (4\%) que presenta la población.

El comportamiento de la resistencia de $M$. tuberculosis en Chile demuestra el éxito del Programa Nacional de Control de Tuberculosis.

\section{Neisseria gonorrhoeae}

La vigilancia de resistencia de este agente se basa en el envío de cepas aisladas en los diferentes centros de salud al centro de referencia del ISP de Chile. Esta vigilancia fue de carácter obligatorio hasta el año 1999 (Tabla 2).

Se ha detectado un creciente aumento en la resistencia tanto a penicilina como a tetraciclina. En el Gráfico 1 se muestra la situación en 1999, en que se puede apreciar el alto porcentaje de susceptibilidad intermedia para estos dos agentes, al igual que se esboza el riesgo de emergencia de resistencia a ciprofloxacina, fármaco de elección en la actualidad.

\section{Haemophilus influenzae}

La vigilancia de resistencia se implementó en el marco de vigilancia de enfermedades invasoras causadas por este agente en población pediátrica y como paso previo a la incorporación de la vacuna anti $H$ influenzae tipo b en el esquema de vacunación nacional. Actualmente se mantiene vigente la norma de envío de toda cepa obtenida de un proceso invasor al centro de referencia del ISP.

$\mathrm{El}$ análisis de la resistencia a este agente se aprecia en el Gráfico 2.

Se aprecia una reducción de la resistencia a ampicilina y cloranfenicol. La situación frente a cefalosporinas de tercera generación es difícil de interpretar y tal vez requiera de un mayor

Tabla 1. Porcentaje de resistencia a agentes antituberculosos en cepas de Mycobacterium tuberculosis*

\begin{tabular}{|c|c|c|c|c|c|c|c|c|c|c|}
\hline Año & $\begin{array}{c}1971 \\
\text { n: } 1.352\end{array}$ & $\begin{array}{c}75 \\
\text { n: } 1.208\end{array}$ & $\begin{array}{c}76 \\
\text { n: } 965\end{array}$ & $\begin{array}{c}77-78 \\
\text { n: } 1.694\end{array}$ & $\begin{array}{c}81 \\
\text { n: } 1.737\end{array}$ & $\begin{array}{c}82 \\
\mathrm{n}: 1.096\end{array}$ & $\begin{array}{c}84-85 \\
\text { n: } 1.070\end{array}$ & $\begin{array}{c}88-89 \\
\text { n: } 1.167\end{array}$ & $\begin{array}{c}95 \\
\mathrm{n}: 920\end{array}$ & $\begin{array}{c}97 \\
\text { n: } 731\end{array}$ \\
\hline Resistencia inicial & 11,4 & 10,1 & 11,7 & 9,6 & 7,9 & 8,7 & 10,2 & 10,4 & 10,8 & 9,2 \\
\hline Resistencia adquirida & 48,5 & 32,3 & 36,5 & 40,3 & 21,2 & 28,9 & 26,5 & 25,4 & 24,0 & 21,2 \\
\hline $\mathrm{n}$ & 803,0 & 588,0 & 334,0 & $1.440,0$ & - & 636,0 & $1.382,0$ & $1.044,0$ & 363,0 & 405,0 \\
\hline
\end{tabular}

Tabla 2. Resistencia a antimicrobianos en cepas de Neisseria gonorrhoeae 1982-1999*

\begin{tabular}{|c|c|c|c|c|c|c|c|c|c|c|}
\hline & 1982 & 84 & 87 & 91 & 94 & 95 & 96 & 97 & 98 & 99 \\
\hline Penicilina & 3,1 & 3 & 4,9 & 19,4 & 29,2 & 14,8 & 37,3 & 39,5 & 25,9 & 34,2 \\
\hline Tetraciclina & 9,1 & 8,3 & 9 & 38,5 & 6,2 & 13,1 & 30 & 22,3 & 27,2 & 37,4 \\
\hline Ciprofloxacina & - & - & - & - & - & - & - & - & - & 0,0 \\
\hline
\end{tabular}




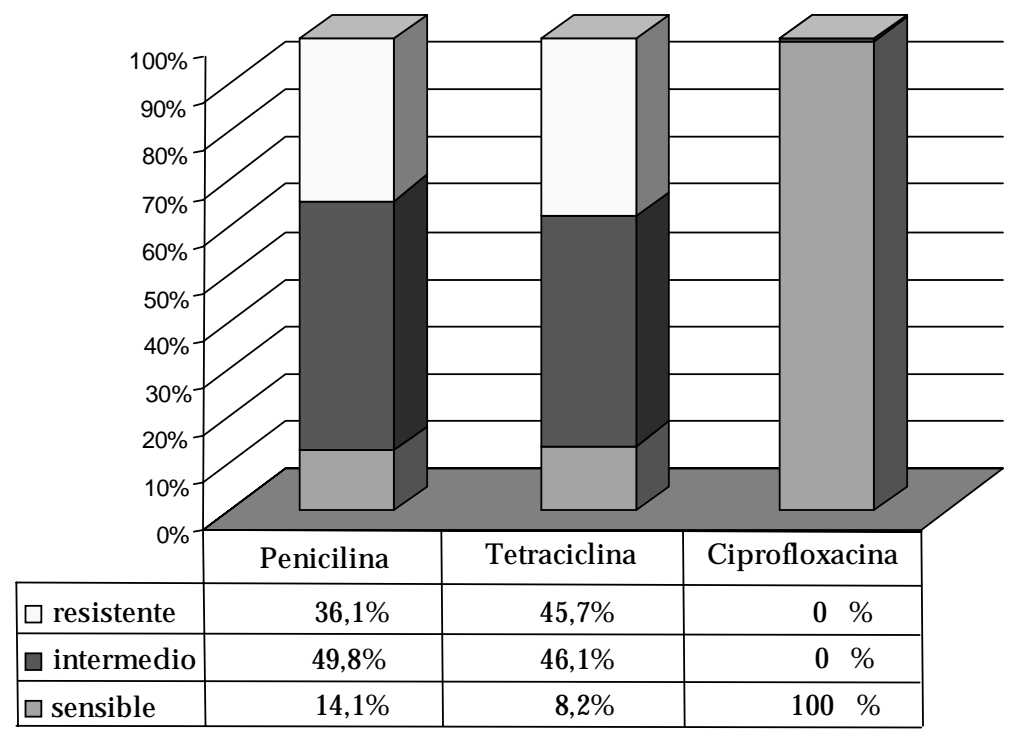

Gráfico 1. Comportamiento frente a antimicrobianos de Neisseria gonorrhoeae año 1999 (n: 219).

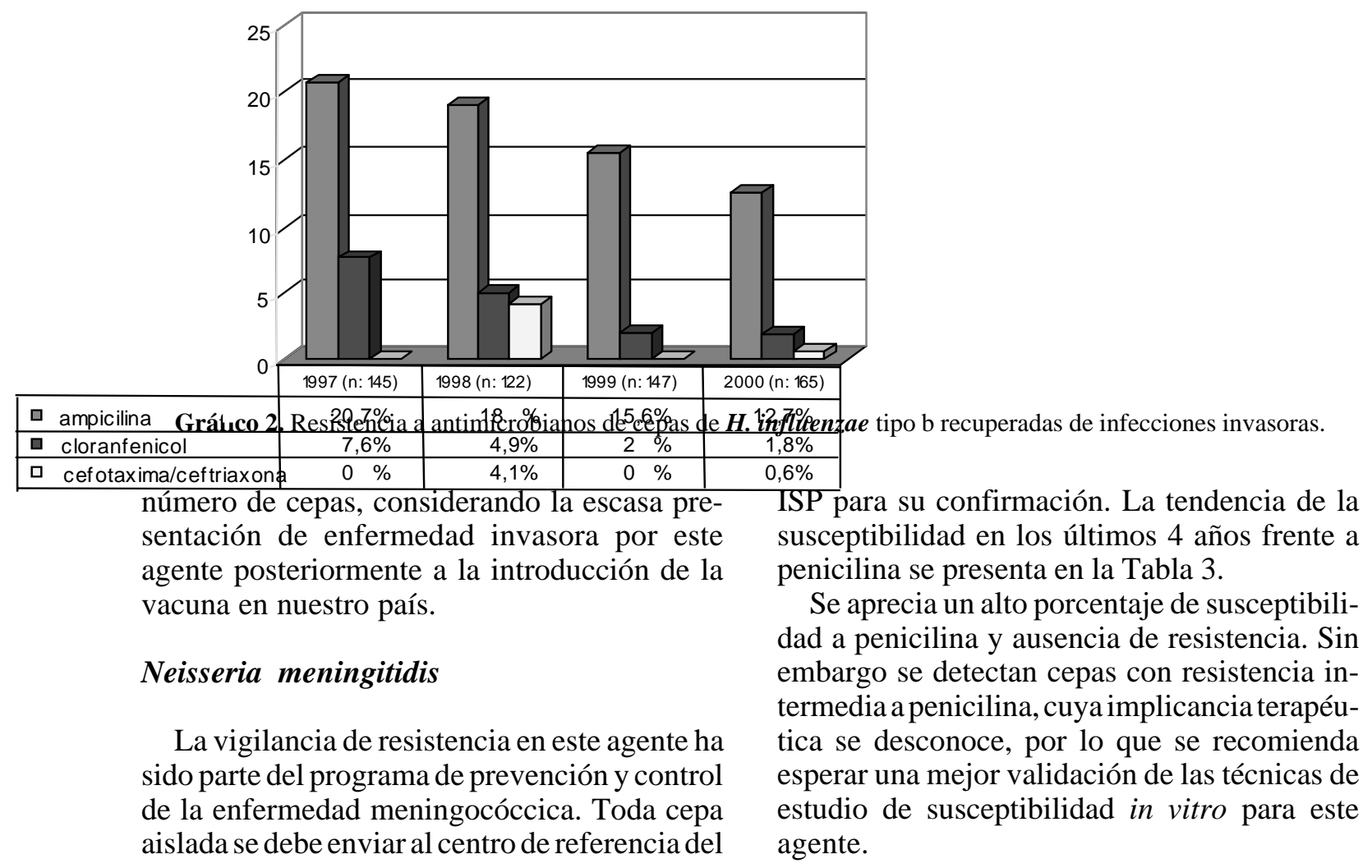


Vigilancia de la resistencia a antimicrobianos - P. González A.

Tabla 3. Resistencia de Neisseria meningitidis a antimicrobianos

\begin{tabular}{|c|c|c|c|c|}
\hline & $\begin{array}{c}1997 \\
\text { (n: 366) }\end{array}$ & $\begin{array}{c}98 \\
\text { (n: 321) }\end{array}$ & $\begin{array}{c}99 \\
\text { (n: 367) }\end{array}$ & $\begin{array}{c}2000 \\
\text { (n: 381) }\end{array}$ \\
\hline Susceptible & $60,1 \%$ & $86 \%$ & $37,9 \%$ & $81,9 \%$ \\
\hline Susceptibilidad intermedia & $39,9 \%$ & $14 \%$ & $62,1 \%$ & $18,1 \%$ \\
\hline Resistente & $0 \%$ & $0 \%$ & $0 \%$ & $0 \%$ \\
\hline
\end{tabular}

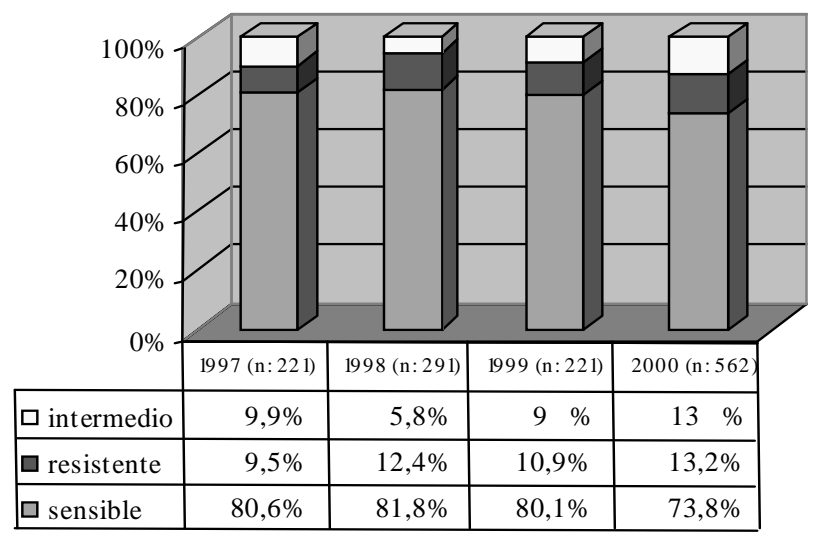

Gráfico 3. Susceptibilidad a penicilina en Streptococcus pneumoniae

Tabla 4. Susceptibilidad de Streptococcus pneumoniae a otros antimicrobianos

\begin{tabular}{lcrrr}
\hline & $\mathbf{1 9 9 7}$ & $\mathbf{9 8}$ & $\mathbf{9 9}$ & \multicolumn{2}{c}{$\mathbf{2 0 0 0}$} \\
$(\mathbf{n : ~ 2 2 1})$ & $(\mathbf{n : ~ 2 9 1 )}$ & $(\mathbf{n : ~ 2 2 1 )}$ & 562) \\
\hline Eritromicina & $5,8 \%$ & $8,5 \%$ & $14,9 \%$ & $10 \%$ \\
Cotrimoxazol & $44,2 \%$ & $39,6 \%$ & $25,5 \%$ & $31,5 \%$ \\
Cloranfenicol & $0 \%$ & $2,8 \%$ & $0 \%$ & $2,5 \%$ \\
Cefotaxima & $4,5 \%$ & $4,8 \%$ & $5 \%$ & $9,3 \%$ \\
& & & & \\
\hline
\end{tabular}

\section{Streptococcus pneumoniae}

La vigilancia de las infecciones invasoras causadas por este agente en población pediátrica se inició en los últimos años. En forma simultánea se inició la vigilancia de resistencia en estas cepas enviadas al centro de referencia del ISP. (Gráfico 3).

En el período estudiado se observó una disminución de cepas susceptibles en base a un incremento tanto de cepas con susceptibilidad intermedia como cepas resistentes.

La situación frente a otros antimicrobianos muestra un considerable aumento de resisten- cia a macrólidos y cefalosporinas de tercera generación (Tabla 4).

\section{Agentes de infecciones intrahospitalarias (IIH) en Chile}

Esta vigilancia, inserta en el programa nacional de prevención y control de IIH se caracteriza por ser obligatoria en todo hospital público y por estar basada en datos obtenidos en cada institución de salud. La recolección de datos se realiza en forma bianual, y está dirigida a evaluar agentes de IIH seleccionados frente a ciertos antimicrobianos. 
Vigilancia de la resistencia a antimicrobianos - P. González A.

Panorama sinóptico de resistencia (\%) en especies de origen nosocomial

\begin{tabular}{llccc}
\hline Especie & Antimicrobiano & $\mathbf{1 9 9 1 - 9 3}$ & $\mathbf{9 4 - 9 6}$ & $\mathbf{1 9 9 9}$ \\
\hline S. aureus & cloxacilina & 34,5 & 48,4 & 56 \\
A. baumannii & ampicilina/ & & & 38 \\
& sulbactam & 8,1 & 10,5 & 29 \\
P. aeruginosa & ceftazidima & 22 & 10,8 & \\
K. pneumoniae & cefotaxima & 22,4 & 34,5 & \\
\hline
\end{tabular}

En los dos últimos años se ha integrado al sistema de vigilancia el estudio de portación de Enterococcus resistente a vancomicina. Esta consiste en un estudio de prevalencia mensual de portación rectal de este agente en pacientes hospitalizados de alto riesgo.

En resumen, el control la resistencia a antimicrobianos en el futuro se vislumbra como uno de los desafíos de la medicina actual y futura, pues a la situación actual se agregó nuevas problemáticas como la diseminación de la resistencia desde instituciones de salud hacia la comunidad, al igual que el impacto de la globalización que impide la contención geográfica que muchas veces nos ha protegido.

Por esto como país debemos aunar esfuerzos para detectar situaciones problemáticas y evaluar las diferentes estrategias de prevención. Como un pilar fundamental es necesario disponer de sistemas de vigilancia de resistencia antimicrobiana que permitan, a través de una metodología válida, la detección oportuna de los cambios de tendencia.

Cabe señalar que una vigilancia no tiene valor si no es analizada y difundidos sus resultados en forma oportuna de manera tal que permita tomar conductas preventivas y de control.

Entre las tareas a corto plazo se requiere

- incentivar el análisis de vigilancia de resistencia a nivel hospitalario

- Establecer redes de vigilancia que apunten a ciertos problemas ej: UCI, centros centinelas, etc.
- Implementar estrategias de control de acuerdo a las diferentes realidades.

Por último, el o los sistemas de vigilancia que se implementen deben mantener presente el objetivo final de ésta, esto es permitir una adecuada detección de la emergencia de resistencia, para lo cual deben asegurar un acucioso análisis de los datos y una oportuna difusión de los resultados a la comunidad médica.

"La resistencia a antimicrobianos como un fenómeno per se no es una sorpresa.

Tampoco es algo nuevo. Aún así, de nuevo nos preocupa ya que la resistencia crece aceleradamente, mientras que las

herramientas que el mundo cuenta para combatirla disminuyen en poder y número."

Joshua Lederberg, Premio Nobel

Agradecimientos. A TM Soledad Prat, Laboratorio de Referencia ISP, por datos de vigilancia en Haemophilus influenzae, Streptococcus pneumoniae y Neisseria meningitidis. A aTM Aurora Maldonado, Laboratorio de Referencia ISP, por datos de vigilancia en Neisseria gonorroheae.

\section{BIBLIOGRAFÍA}

1.- VALENZUELA P, PIFFARDI S, LEPE R, VELASCO M. Panorama actualizado de la resistencia a fármacos antituberculosos. Chile 1998. Rev Chil Infect 1998; 15 (4): 225-33.

2.- Informes periódicos ISP.

Correspondencia a:

Patricia González Aguirre

E-mail:patgonzalez@hotmail.com 